\title{
Identification of Barely Visible Impact Damages on a Stiffened Composite Panel with a Probability-based Approach
}

\author{
MARIA MOIX BONET, BENJAMIN ECKSTEIN, \\ RICHARD LOENDERSLOOT and PETER WIERACH
}

\begin{abstract}
A probability-based damage detection algorithm has been implemented in order to identify barely visible impact damages in two composite stiffened panels by means of Acousto Ultrasonics (AU). A modification of RAPID (reconstruction algorithm for probabilistic inspection of defects) has been implemented to adapt the algorithm to the current structures and transducer networks. An improvement of $40 \%$ in the localization accuracy is obtained with the new algorithm.
\end{abstract}

\section{INTRODUCTION}

Carbon-Fiber Reinforced Polymer (CFRP) is gaining ground as preferred material for the construction of aircraft structures. Despite its multiple advantages, composite laminates are prone to suffer barely visible impact damages (BVID). The impact damage proneness is currently addressed with a damage tolerant design and an increase of Non-Destructive Inspections (NDI). Structural Health Monitoring (SHM) targets to be used as a complement of NDI, enabling a decrease in maintenance efforts. Acousto Ultrasonics, an SHM technique, is based on a permanently installed piezoelectric transducer network, which actuates and receives ultrasonic guided waves to provide information concerning the structural integrity [1]. A reliable damage detection procedure is under development so that AU represents a feasible alternative to the currently used NDI in aircraft structures.

A probabilistic approach termed RAPID (reconstruction algorithm for probabilistic inspection of defects) has been reported to be effective in detecting damage in structures with complex geometries. Zhao et al. [2] detected and localized rivet cracks and corrosion on an aircraft aluminum wing, Wu et al.[3] used the RAPID with a composite panel with bonded T-stringers, combining several frequencies for an improved damage assessment, and Wang et al. [4] identified a through-thickness hole in a composite stiffened panel.

M. Moix Bonet and P. Wierach, German Aerospace Center, Lilienthalplatz 7, 38108 Braunschweig, Germany

R. Loendersloot, University of Twente, P.O. Box 217, 7500AE, Enschede, The Netherlands

B. Eckstein, Airbus Group Innovations, Airbus-Allee 1, 28199 Bremen, Germany 
The RAPID is nevertheless not optimal for the monitoring of large surfaces using transducers regularly and sparsely located throughout the structure. The presented work reports the results obtained with a modified RAPID for the damage identification on two stiffened composite panels, in which realistic BVID have been introduced.

\section{EXPERIMENTAL SETUP}

Two identical composite structures have been manufactured within the EU FP7 project "SARISTU". Each panel comprises 3 co-bonded omega stringers and 40 cobonded piezoelectric transducers; the first panel is equipped with SMARTLayer ${ }^{\circledR}$ transducers and the second with DuraAct ${ }^{\mathrm{TM}}$ transducers. The skin has a constant thickness, a quasi-isotropic $2 \mathrm{~mm}$ layup and a slight curvature. A schematic of the panel design with a detail of the stringer profile is shown in Figure 1.

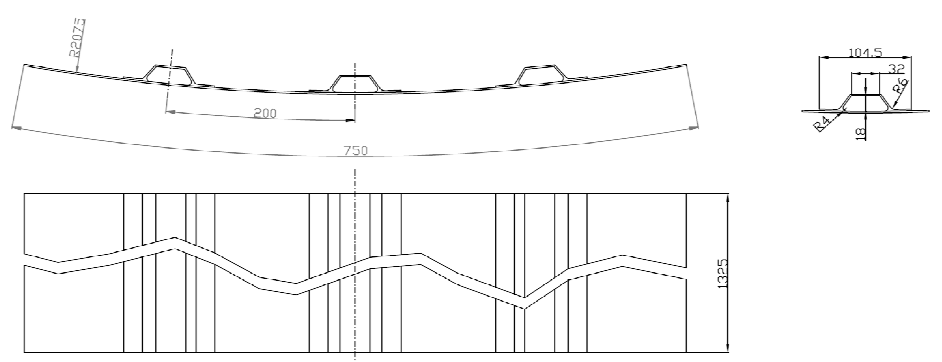

Figure 1: Design of the CFRP panel without SHM network [5]

The impact campaign consists in several impacts introduced from the outer skin in each panel with energies between the 20 Joules and the 34 Joules applied by means of a hemispherical projectile with a diameter of $25 \mathrm{~mm}$. This amount of energy targets barely visible impact damages and debondings on the described structures. The area of the introduced damages measures between $518 \mathrm{~mm}^{2}$ and $690 \mathrm{~mm}^{2}$ : The diameter of a round damage with this area would measure $27.6 \pm 2 \mathrm{~mm}$. In order to determine the performance of the damage identification algorithms, the damages have been measured with traditional NDI methods. The geometry, SHM-network and impact positions in one of the structures, as well as two of the defects inspected with NDI are depicted in Figure 2.
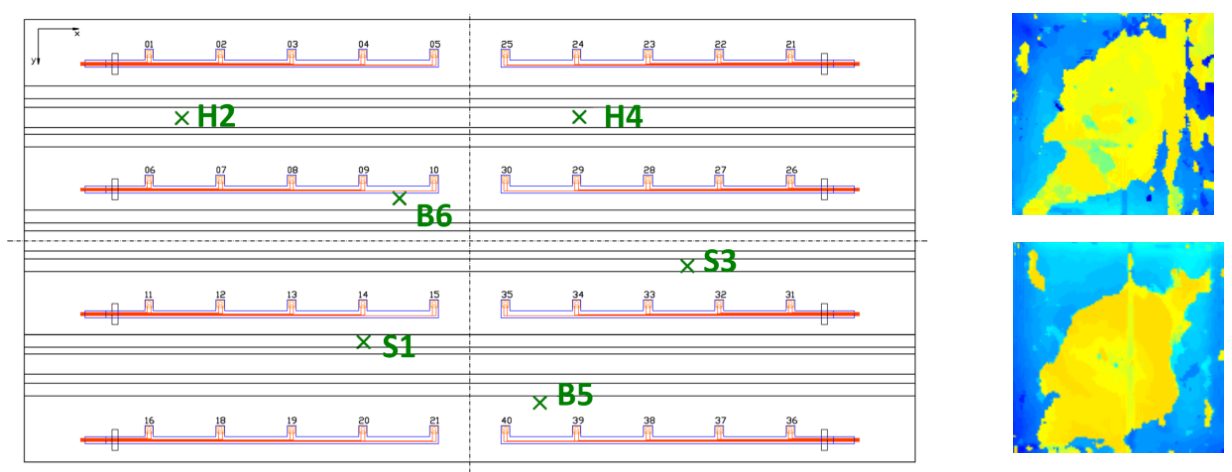

Figure 2 Sketch of the structure and SMARTLayer ${ }^{\circledR}$ network with impact locations (left) and ultrasound scan of the damages H2 (right-up) and H4 (right-down) 
The damage effect on the guided wave propagation depends on the relative damage position within the structure. Three damage types are therefore considered:

- H-type damages: located under a stringer head

- S-type damages: located under a stringer foot and targeting a debonding

- B-type damages: positioned on the skin, between transducers

\section{METHODOLOGY}

The damage assessment is performed by means of a probability-based methodology along with a graphical user interface developed within the study and described in detail in [5].

The usual transducer network configuration employed to identify damage with the RAPID algorithm consists of the transducer network located around the damage (see [2-4]). This study aims at damage identification in any part of the structure with a transducer network regularly distributed on the structure. With the given conditions, the original RAPID algorithm has the tendency to show high damage probability on the transducer positions, since the path density is rather high on those areas.

A first approach ignores the damage probability in the transducers vicinity. The approach is reported in [6] and used as starting point in the current work. The location accuracy improves substantially, but the probability distribution function still increases towards the sensors positions and damages cannot be located on the sensor surroundings. A second approach, presented in the current paper, modifies the area of influence of each path introducing a coefficient $\alpha$ and optimizing both $\alpha$ and $\beta$, the scaling factor. The algorithm modification aims at a more homogeneous probability distribution function, avoiding the high probability near the transducers.

To calculate the damage probability with RAPID, first, a grid is defined, overlaying the panel. The damage intensity $I(x, y)$ at each grid point $(x, y)$ is calculated, by assuming a certain area of influence of the actuator-sensor paths. The damage intensity if defined as:

$$
I(x, y)=\sum_{k=1}^{N_{p}}\left(1-\rho_{k}\right)\left(\frac{\beta-R(x, y)}{\beta-1}\right)
$$

with $\rho_{k}$ being the damage index of the $k^{\text {th }}$ actuator-sensor path, $N_{p}$ the number of paths, $\beta$ the scaling factor determining the area of influence and $R(x, y)$ defined as:

$$
R(x, y)=\left\{\begin{array}{cc}
\frac{\sqrt{\left(x-x_{i}+\alpha\left(x_{i}-x_{j}\right)\right)^{2}+\left(y-y_{i}+\alpha\left(y_{i}-y_{j}\right)\right)^{2}}+\sqrt{\left(x-x_{j}+\alpha\left(x_{j}-x_{i}\right)\right)^{2}+\left(y-y_{j}+\alpha\left(y_{j}-y_{i}\right)\right)^{2}}}{\sqrt{\left(x_{i}-x_{j}-2 \alpha\left(x_{i}-x_{j}\right)\right)^{2}+\left(y_{i}-y_{j}-2 \alpha\left(y_{i}-y_{j}\right)\right)^{2}}} & \text { for } R(x, y)<\beta \\
\beta & \text { for } R(x, y) \geq \beta
\end{array}\right.
$$

where $\left(x_{i}, y_{i}\right)$ and $\left(x_{j}, y_{j}\right)$ indicate the locations of transducer $i$ and $j$ respectively The coefficient $\alpha$ is introduced to modify the location of the probability distribution function. The effects of $\alpha$ and $\beta$ on the distribution function for a single actuatorsensor pair are depicted in Figure 3. 


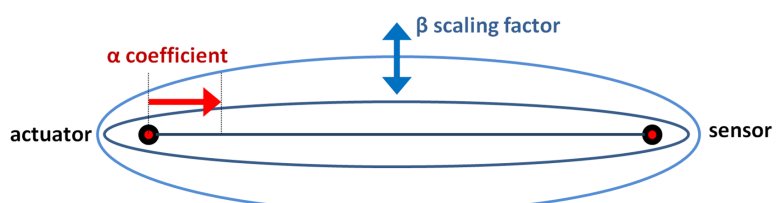

Figure 3: Elliptical distribution function for a single path with effects of $\alpha$ and $\beta$

The damage assessment is carried out through a comparison between a baseline, acquired with the structure in a pristine state, and the current signal, acquired in a normally unknown state of the structure. A change in the structure characteristics translates into a variation in the acquired signal, which has to be interpreted properly. In this paper it is assumed that the changes on the signal are only dependent on the appearance of damage.

The comparison between the two signals is quantified with a Damage Index (DI). In the developed damage identification tool fourteen DI are implemented. The DI suitability to detect damage applied to the current structure and damage type has been previously investigated in [6]. The four most successful DI are employed in the current paper and their corresponding formulas are presented in Table 1.

TABLE 1: DAMAGE INDEX INCLUDED IN DAMAGE IDENTIFICATION SOFTWARE [5]

\begin{tabular}{llcl}
\hline Method Name & Abbr. & Mathematical Formula \\
\hline Correlation coefficient & CC & $\rho=\frac{\sum_{i=1}^{N} S_{H, i} S_{D, i}-\sum_{i=1}^{N} S_{H, i} \sum_{i=1}^{N} S_{D, i}}{\sqrt{\sum_{i=1}^{N} S_{H, i}^{2}-\left(\sum_{i=1}^{N} S_{H, i}\right)^{2}} \cdot \sqrt{\sum_{i=1}^{N} S_{D, i}^{2}-\left(\sum_{i=1}^{N} S_{D, i}\right)^{2}}}$ \\
Signal Sum of Squared Differences & SSSD & DI $=1-\frac{\sum\left(S_{H}-S_{D}\right)^{2}}{\sum_{H}^{2}}$ & $\rho=\frac{1}{D I+1}$ \\
$\begin{array}{l}\text { Discrete Wavelet Transform } \\
\text { Approximation Coefficients }\end{array}$ & DWTC & $D I=1-\frac{\sum\left(\mathbf{D W T}\left[S_{H}\right]-\mathbf{D W T}\left[S_{D}\right]\right)^{2}}{\sum \mathbf{D W T}\left[S_{H}\right]^{2}}$ & $\rho=\frac{1}{D I+1}$ \\
$\begin{array}{l}\text { Ratio of Covariance Matrix } \\
\text { Eigenvalues }\end{array}$ & $\mathrm{RCME}$ & $\rho=1-\frac{\lambda_{2}}{\lambda_{1}}$ & \\
\hline
\end{tabular}

The performance of the developed methodology is evaluated through comparison with the position and size of the damages observed with traditional NDI methods. An example of the analysis is depicted in Figure 4. A table with the results obtained with NDI and AU is presented on the right. The damage assessment is performed with signals acquired in a frequency range between $60 \mathrm{kHz}$ and $90 \mathrm{kHz}$; the damage intensity plot is shown on the left.

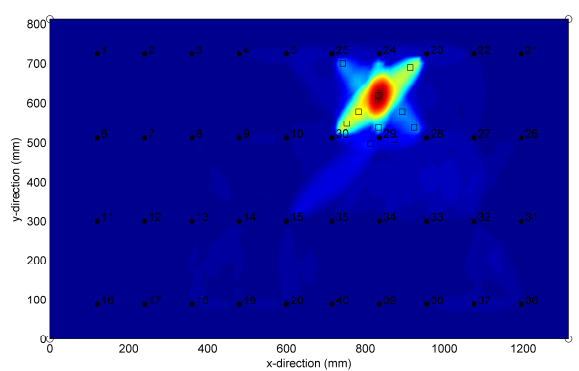

\begin{tabular}{|c|c|c|}
\hline $\mathbf{X}(\mathbf{m m})$ & $\mathbf{Y}(\mathbf{m m})$ & Area $\left(\mathbf{m m}^{2}\right)$ \\
\hline & \multicolumn{3}{|c|}{ Measured with NDI } \\
\hline 835 & 618 & 574.06 \\
\hline Detected with $60-90 \mathrm{kHz}-$ threshold $95 \%$ \\
\hline 833.2 & 621.5 & 586.9 \\
\hline
\end{tabular}

Figure 4: SARISTU Damage detection of damage H4 with the NDI and SHM results 


\section{RESULTS \& DISCUSSION}

\section{Parameter Optimization}

An initial research is carried out to optimize the $\alpha$-coefficient and $\beta$ scaling factor. The aim of the damage identification algorithm in the current scenario is to locate damage in any part of the monitored structure. Therefore, given an equal DI for every path due to noise, ideally the damage probability shall be uniform throughout the surface.
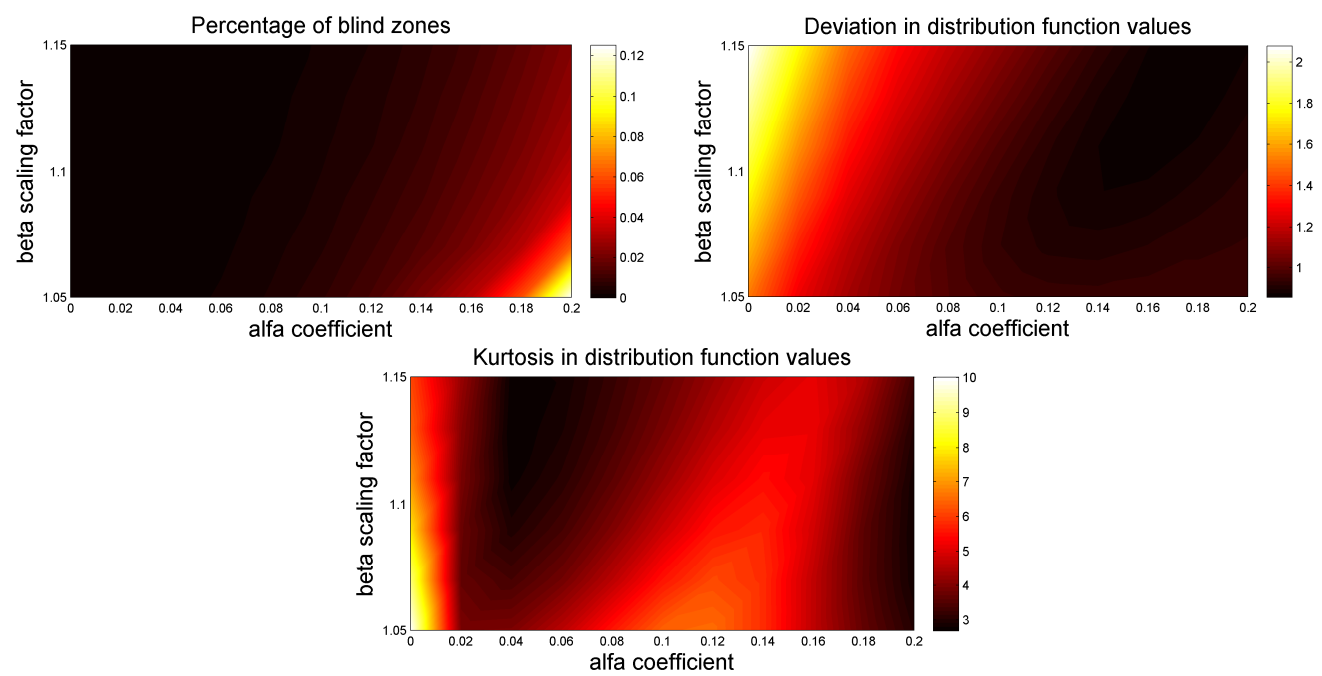

Figure 5: Blind zones (top-left), deviation (top-right) and kurtosis (bottom) of probability distribution function for the given path arrangement

The $\alpha$-coefficient and $\beta$ scaling factor have been chosen according to three criteria, which pursue a uniform probability distribution function. For the calculation the global damage intensity distribution function $I(x, y)$ with equal DI for all paths has been employed:

- Blind zones: The surface percentage, which the algorithm does not cover given a combination of $\alpha$ and $\beta$ (see Figure 5- top-left).

- Deviation of $I(x, y)$ (see Figure 5 - top-right).

- Kurtosis of $I(x, y)$ : Indicates the shape, the "peakedness", of the distribution function (see Figure 5- bottom).

An optimal $\alpha-\beta$ pair should give the lowest values for the three criteria. A compromise has to be reached, since the tendencies of the three characteristics have differing trends, as presented in Figure 5. A coefficient $\alpha=0.08$ and scaling factor $\beta=1.07$ are selected for the ensuing damage identification analysis.

The original distribution function $R(x, y)$ with $\alpha=0.0, \beta=1.05$ and omitted transducer surrounding areas is depicted in Figure 6 (left), while the function with the optimized parameters is plotted in Figure 6 (right). A more homogeneous probability throughout the structure can be recognized on the right, avoiding the high probability on the transducers horizontal line as well as the peaks observed near the transducers. The new configuration avoids the damage localization shift towards transducer positions while improving sensitivity on the areas where lower transducer density and stringers hinder damage detection. 

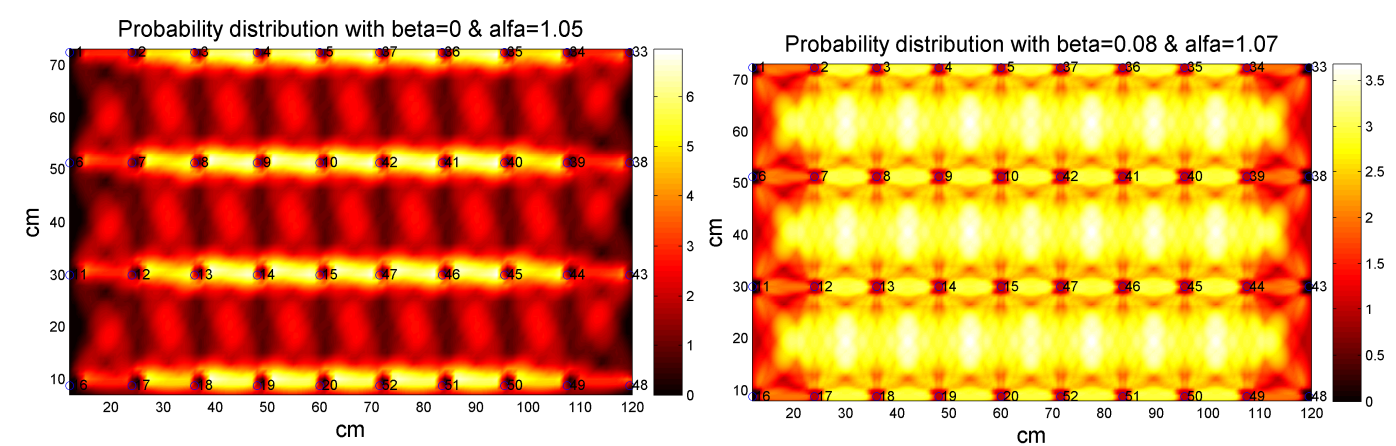

Figure 6: Probability distribution function with original (left) and optimized parameters (right)

\section{Damage Identification}

The analysis shown in Figure 4 is reproduced for the four selected DIs described in the methodology and eight BVID. Two parameters are used to quantify the performance of the identification algorithm: the damaged-to-undamaged probability ratio and the damage localization error. The first consists of a ratio to evaluate the sensitivity of the DI in relation to the noise level. The ratio compares the maximal damage probability obtained with the identification algorithm when two baselines (no presence of damage) are compared and the maximal probability acquired when damage is present. The damage localization error, the second parameter, compares the damage locations in the $x$ and $y$ coordinates obtained with the traditional NDI methods and the ones obtained with the damage identification algorithm.

A combination of the four DIs is employed, obtaining a more reliable and sensitive identification algorithm. From the selected DIs, the maximal DI value is taken for each path as input for the diagnostic imaging algorithm. The outcome is an enhanced ratio between the damage probability obtained in damaged and pristine states, as depicted in Figure 7.

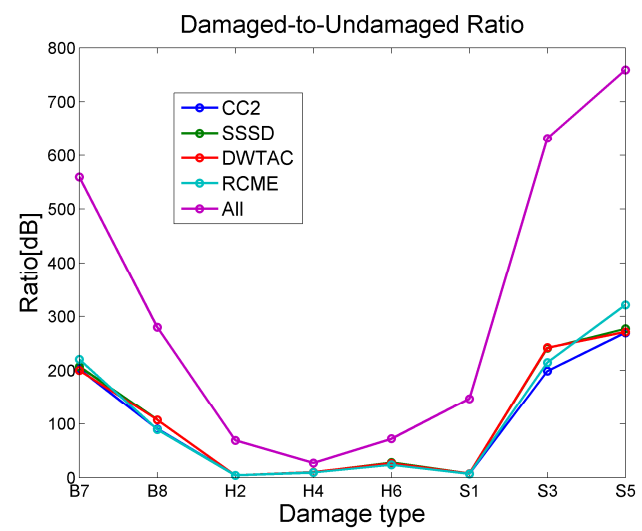

Figure 7: Amplitude ratio between damaged and pristine signals
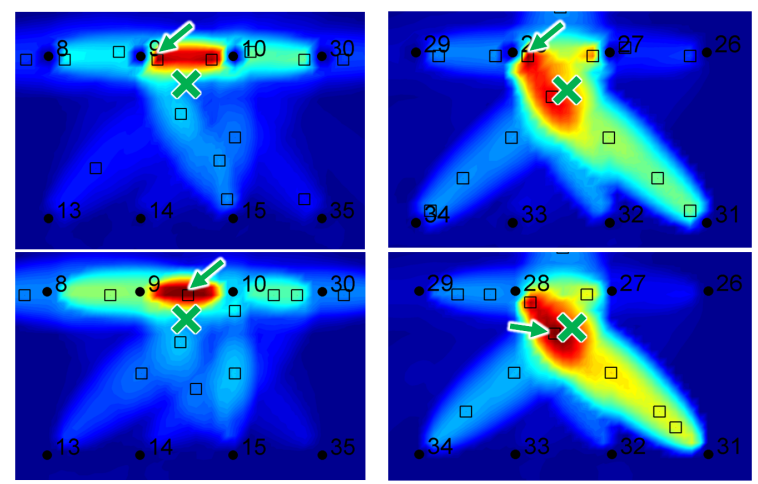

Figure 8: Damage identification before (top) and after (down) RAPID modification for damages B7 (left) and S5 (right) showing real (cross) and detected (arrow) damage location 
Figure 9 (left) presents the averaged absolute error of the damage localization using each DI separately and afterwards all DIs combined. Several noteworthy observations can be mentioned from the localization accuracy. Firstly, a slight decrease of $8 \%$ in the error made can be observed when all DIs are used. Secondly, the bars in blue indicate the averaged localization error made with the initial configuration and the ones in green depict the error with optimized $\alpha-\beta$ values: The location accuracy improves a $40 \%$ with the optimized configuration. Finally, the error in the $x$-coordinate of the location is twice as low as that of the $y$-coordinate. This outcome is caused by several reasons. On the one side, the density of transducers in the $x$-direction is 1.7 times higher than in the $y$-direction; the transducer density is a determining parameter for the localization accuracy. On the other side, the geometry of the structure has an influence on the damage detection capability of the algorithm: Between two adjacent transducers in the $y$-direction, there is always an Omega stringer present.
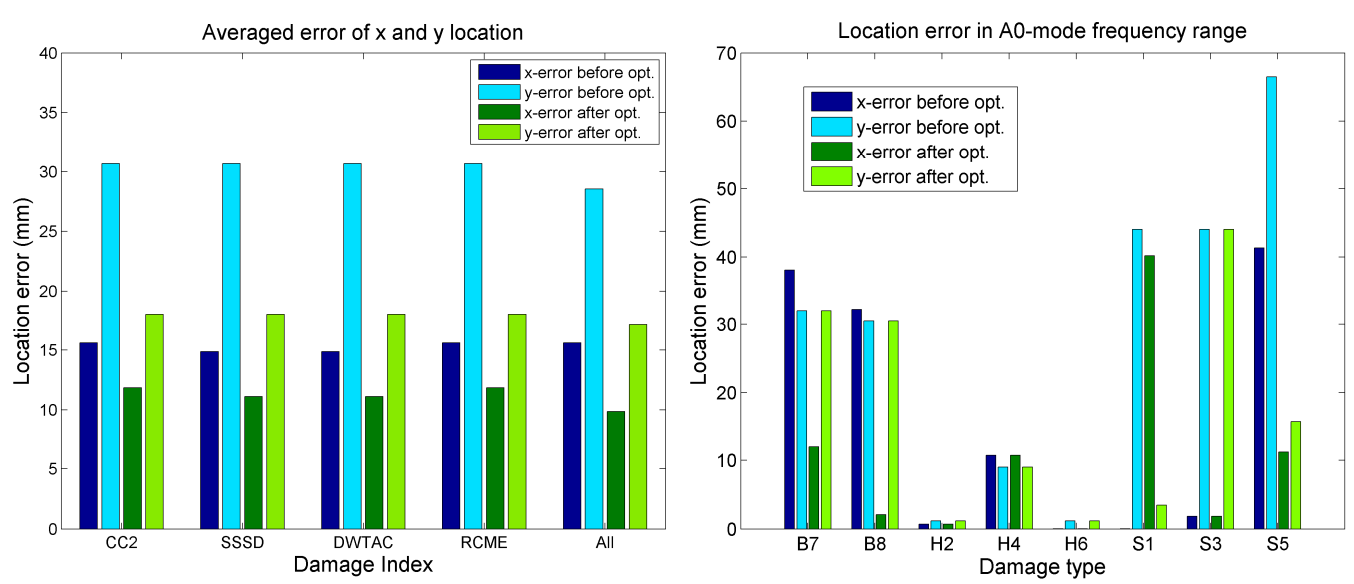

Figure 9: Location error before and after optimization as a function of the DIs (left) and damage types (right)

In Figure 9 (right) the error in damage location obtained with the DIs combined is plotted as a function of the damage type. The error as well as the localization improvement due to the new configuration highly depends on the damage type:

- The algorithm can accurately locate H-type damages, with a maximum of $13 \mathrm{~mm}$ error, and does not show improvement after algorithm optimization.

- The localization of the B-type improves in accuracy in the $x$-coordinate while the $y$-coordinate error remains constant. The B-type damage is mainly detected with horizontal paths; the $y$-location is therefore erroneously estimated to be located at the transducer $y$-coordinate (see Figure 8 left). The error in the $x$-coordinate is due to the tendency of the identification algorithm to show higher amplitude near the transducers: This tendency is corrected with the modified algorithm. The damage diagnostic of $\mathrm{B} 8$ with both configurations is presented in Figure 8 (left).

- The $y$-location of the S-type damage, i.e. the debonding between skin and stringer, is challenging for the algorithm. The cause lies again in the tendency 
to shift the maximal damage probability towards the transducer positions. This tendency is corrected with the algorithm modification, as shown in Figure 8 (right).

\section{CONCLUSION}

The paper exploits the possibilities of the developed damage identification methodology by applying it to the damage assessment of two stiffened composite panels. The damage identification algorithm is capable of identifying the $100 \%$ of the introduced barely visible impact damages. It is possible to estimate the location of the damage with an averaged error of $10 \mathrm{~mm}$ in the $x$-coordinate and $17 \mathrm{~mm}$ in the $y$ coordinate.

Two main modifications are carried out in comparison with the original RAPID: the use of DI other than the correlation coefficient, and the adjustment of the influence area of each path by means of the introduction of the $\alpha$-coefficient. On the one side, the fusion of several DIs results in an enhanced sensitivity and accuracy, when compared to the damage assessment with a single DI. On the other side, the introduction of the $\alpha$-coefficient improves the damage localization for the given composite structure and transducer network by $40 \%$.

The amount of data from real damages is rather limited to draw solid conclusions. The data generated during the impacting campaign of a generic door surrounding aircraft structure with 584 transducers and the introduction of 104 BVID will be used to further evaluate and optimize the algorithm.

\section{ACKNOWLEDGMENTS}

The research leading to these results has received funding from the European Union's Seventh Framework Programme for research; technological development and demonstration under grant agreement no 284562.

\section{REFERENCES}

1. Giurgiutiu, V. 2005. "Tuned Lamb wave excitation and detection with piezoelectric wafer active sensors for structural health monitoring," J. Intell. Matls. Sys. \& Struct., pp. 291-305

2. Zhao, X., Gao, H., Zhang, G., Ayhan, B. Yan, F., Kwan, C., Rose, J.L. 2007. "Active health monitoring of an aircraft wing with embedded piezoelectric sensor/actuator network: I. defect detection, localization and growth monitoring," Smart Materials and Structures 16, 1208-1217

3. Wu Z., Liu K., Wang Y. and Zheng Y. 2014. „Validation and evaluation of damage identification using probability-based diagnostic imaging on a stiffened composite panel," $J$. Intell. Matls. Sys. \& Struct., 1045389X14549873

4. Wang D., Ye L., Lu Y., Su Z. 2008. „Probability of the presence of damage estimated from an active sensor network in a composite panel of multiple stiffeners," Composites Science and Technology 69 (2009) 2045-2063

5. Loendersloot R., Büthe I., Michaelidos P., Moix-Bonet M. 2015. "Damage Identification in Composite Panels - Methodologies and Visualisation" SARISTU Final Conference, Moscow, Russia.

6. Moix-Bonet M., Loendersloot R., Bach M., Wierach P. 2015. "Damage Assessment in Composite Structures based on Acousto Ultrasonics - Evaluation of Performance," SARISTU Final Conference, Moscow, Russia. 